\title{
Commentary: Postoperative Pain Management Strategies in Hip Arthroscopy
}

\author{
Collin LaPorte ${ }^{1,2 *}$, Michael D Rahl ${ }^{2}$, Olufemi R Ayeni ${ }^{3}$ and Travis J Menge ${ }^{1,2}$ \\ ${ }^{1}$ Spectrum Health Medical Group Orthopedics \& Sports Medicine, Grand Rapids, MI, USA \\ ${ }^{2}$ Michigan State University College of Human Medicine, Grand Rapids, MI, USA \\ ${ }^{3}$ Division of Orthopaedic Surgery, McMaster University, Hamilton, ON, Canada
}

${ }^{\star}$ Corresponding author: Collin LaPorte, Spectrum Health Medical Group Orthopedics \& Sports Medicine, Michigan State University College of Human Medicine, Grand Rapids, MI, USA

Received: February 18, 2021; Accepted: February 23, 2021; Published: March 01, 2021

\begin{abstract}
Hip arthroscopy is a rapidly growing field due to its significant diagnostic and therapeutic value in treating a variety of hip disorders. Due to the lack of standardized protocol for pain management in these patients, adequate control of postoperative pain continues to be challenging. Several techniques have been employed to find a regimen that is effective at reducing postoperative pain, narcotic consumption and cost to the patient and healthcare system. The purpose of this article is to provide a review of important conclusions from the previous paper "Postoperative Pain Management Strategies in Hip Arthroscopy" and report on possible implications of the article.

Recent literature supports the use of a multi-modal approach to managing postoperative pain in patients undergoing hip arthroscopy. When a pre-and postoperative analgesic regimen is used in combination with peripheral nerve block or intraoperative anesthetic injection, patients experience less pain and postoperative narcotic consumption. Postoperative pain scores and opioid consumption are similar between the different techniques. However, postoperative complications are less in those receiving Intra-Articular (IA) injection or Local Anesthetic Infiltration (LAI) compared to peripheral nerve blocks.

Recent studies suggest that intraoperative techniques such as IA injection or LAI used in conjunction with a pre-and postoperative analgesic regimen may be the safest and most effective multi-modal strategy for reducing postoperative pain in these patients. In addition, omitting the use of peripheral nerve block may lead to decreased anesthesia procedural fees and operating room turnover time, resulting in decreased cost to the patient and increased efficiency of the facility.
\end{abstract}

\section{Introduction}

Hip arthroscopy is gaining popularity among orthopedic surgeons due to its significant diagnostic and therapeutic value in the management of common hip disorders such as Femoroacetabular Impingement (FAI), labral pathology, loose body, snapping hip, septic arthritis, synovial disorders and gluteus tendon tears. Despite the recent increased prevalence of hip arthroscopy to treat hip pathology, postoperative pain management in these patients continues to be challenging for orthopedic surgeons. Unfortunately, there is a lack of standardized protocols for postoperative pain management in hip arthroscopy, likely due to the paucity of comparative high-quality studies exploring the efficacy of different techniques.

The purpose of Postoperative Pain Management Strategies in Hip Arthroscopy [1] was to provide an up to date comprehensive review of current literature regarding postoperative pain management techniques in patients undergoing hip arthroscopy. In addition, it provides a source that orthopedic surgeons can reference to determine which hip arthroscopy pain management technique, or combination thereof, is best for their practice and patients. The purpose of this commentary is to provide a rapid review of the most important conclusions of Postoperative Pain Management Strategies in Hip Arthroscopy [1] and report on possible implications of the article.

\section{Important Conclusions}

Several recent studies have proven the effectiveness of oral medications such as acetaminophen, gabapentin and cyclobenzaprine for management of pain in patients undergoing major orthopedic surgery [2-4]. However, these drugs have not been extensively studied in patients undergoing hip arthroscopy. In contrast, Celecoxib has been extensively studied in hip arthroscopy and has proven to be an efficacious oral analgesic and nonsteroidal anti-inflammatory (NSAID) due to its high oral bioavailability, rapid absorption, and selective cyclooxygenase (COX)-2 inhibition [5]. In two randomized controlled trials, preoperative celecoxib resulted in significantly lower Visual Analogue Scale (VAS) pain scores at 1, 12 and 24 hours postoperatively compared to placebo $[5,6]$. In addition, patients receiving celecoxib also spent less time in the Post-Anesthesia Care Unit (PACU) compared to placebo [5,6].

There has been growing interest surrounding the use of peripheral nerve blocks such as Lumbar Plexus Block (LPB), Femoral Nerve Block (FNB) and Fascia Iliaca Block (FIB) in the management of postoperative pain in patients undergoing hip arthroscopy. Patients undergoing LPB had statistically, but not clinically significant lower VAS pain scores in the PACU compared to those with general anesthesia only, or placebo $[7,8]$. However, patients receiving LPB 
required less postoperative narcotics, anti-emetics and ketorolac than the control group [7]. Patients who receive FNB tend to require less intraoperative and postoperative narcotics compared to those who receive general anesthesia alone or placebo $[9,10]$. Patient reported pain scores were lower in patients receiving FNB compared to general anesthesia alone, however time in PACU was higher in those receiving FNB [10]. Importantly, patient satisfaction was higher and time to discharge was lower in patients receiving FNB compared to those who received IV morphine for pain [11]. Patients who received FIB during hip arthroscopy had fairly good overall pain scores in PACU $(3.85 / 10)$ [12].

Although peripheral nerve blocks are effective at reducing postoperative pain, they are not without complication. Peripheral nerve blocks have the potential for intravascular injection, iatrogenic nerve injury, postoperative falls, infection, hematoma and rebound pain after discharge [13-15]. In addition, peripheral nerve blocks require specialized equipment and an anesthesiologist to perform them, resulting in increased cost to the patient and hospital.

When compared to FNB, local anesthetic Intra-articular (IA) injections have proven to be just as effective. Child et al. [14] reported no significant difference in patient reported pain scores at 1, 3 and 6 weeks postoperatively compared to FNB. Importantly, the occurrence of postoperative falls in the IA injection group was significantly lower ( 5 vs $19, \mathrm{p}<0.001$ ). There was also a lower rate of postoperative peripheral neuritis in the IA injection group compared to the FNB group ( 2 vs $26 \mathrm{p}<0.001)$. Therefore, IA injection with local anesthetic provides a valuable alternative to FNB for postoperative pain control, due to significantly less complications associated with the procedure and similar pain scores as FNB. In patients who received preoperative celecoxib with acetaminophen plus IA injection with morphine and clonidine, there was a significant reduction in postoperative narcotic consumption in PACU compared to patients who received oral analgesics only. However, pain scores were similar between the two groups and there was no significant difference in time to discharge [16]. Therefore, an IA injection with clonidine and morphine may reduce complications associated with postoperative opioid consumption such as respiratory depression and dependency, while providing adequate analgesia to patients undergoing hip arthroscopy.

Recently, Local Anesthetic Infiltration (LAI) has become popular among orthopedic surgeons as an efficacious alternative to more expensive procedures such as peripheral nerve blocks. In a study conducted by Philippi et al. [17], patients who received intraoperative LAI requested fewer rescue postoperative femoral nerve blocks compared to the non-LAI group. However, there was no significant difference in opioid consumption in PACU between the groups $(\mathrm{p}=0.740)$ [17]. When compared to FIB, patients who received LAI had clinically significant less pain following surgery. In addition, average morphine consumption was twice as low in the LAI group, resulting in less nausea and vomiting compared to the FIB group within 24 hours postoperatively [18]. Interestingly, when LAI was compared with IA injection, patients who received LAI required significantly more rescue medication compared to IA injection group
(2.33 mg vs $0.57 \mathrm{mg}, \mathrm{p}=0.036$ ). However, VAS pain scores were not statistically different between groups at 1 and 2 hours postoperatively [19]. Therefore, LAI is an effective procedure that offers similar pain management outcomes as peripheral nerve blocks, without the risk of intravascular injection, iatrogenic nerve injury, postoperative falls and higher cost to the patient and hospital. Additionally, LAI is performed intraoperatively by the surgeon, without the need for ultrasound guidance which could decrease operating room turnover time.

\section{Implications}

The findings in recent literature support the use of a multi-modal approach to managing postoperative pain in patients undergoing hip arthroscopy. When a multi-modal approach consisting of a pre-and postoperative analgesic regimen combined with peripheral nerve block or intraoperative anesthetic injection is employed, patients experience less pain and postoperative narcotic consumption. Postoperative pain scores and opioid consumption are similar between the different techniques. However, postoperative complications are less in those receiving IA injection or LAI compared to peripheral nerve blocks. In addition, peripheral nerve blocks have the potential for intravascular injection, iatrogenic nerve injury, and require highly trained anesthesiologists resulting in higher costs associated with the procedure. IA injection and LAI are quick procedures performed intraoperatively by the orthopedic surgeon. Use of these intraoperative techniques could increase efficiency of the operating room and decrease cost to the patient and hospital by decreasing turnover time and avoiding anesthesia procedural fees. Therefore, a multi-modal approach consisting of a pre-and post-operative analgesic regimen in combination with IA injection or LAI may be the optimal strategy to manage postoperative pain and increase cost effectiveness of hip arthroscopy.

\section{Conflict of Interest}

Collin LaPorte, Michael Rahl declare no conflicts of interest. Olufemi Ayeni is part of a speaker's bureau for Conmed, outside of the submitted work. Travis Menge reports consulting fees from Smith \& Nephew, and research support/grants from Stryker, DJO, and Smith \& Nephew, outside of the submitted work.

\section{References}

1. LaPorte C, Rahl MD, Ayeni OR, Menge TJ (2019) Postoperative Pain Management Strategies in Hip Arthroscopy. Current Reviews in Musculoskeletal Medicine 12: 479485. [crossref]

2. Schug SA, Sidebotham DA, McGuinnety M, Thomas J, Fox L (1998) Acetaminophen as an Adjunct to Morphine by PatientControlled Analgesia in the Management of Acute Postoperative Pain. Anesthesia \& Analgesia 87: 368-372. [crossref]

3. Han C, Li X, Jiang H, Ma JX, Ma XL (2016) The use of gabapentin in the management of postoperative pain after total hip arthroplasty: a meta-analysis of randomised controlled trials. Journal of Orthopaedic Surgery and Research 11: 79. [crossref]

4. Witenko C, Moorman-Li R, Motycka C, Duane K, Hincapie-Castillo J, et al (2014) Considerations for the appropriate use of skeletal muscle relaxants for the management of acute low back pain. P \& T: a peer-reviewed. Journal for Formulary Management 39: 427-435. [crossref]

5. Kahlenberg CA, Patel RM, Knesek M, Tjong VK, Sonn K, et al. (2017) Efficacy of Celecoxib for Early Postoperative Pain Management in Hip Arthroscopy: A Prospective Randomized Placebo-Controlled Study. Arthroscopy: The Journal of Arthroscopic and Related Surgery 1180-1185. [crossref] 
6. Zhang Z, Zhang Z, Zhu W, et al. (2014) Efficacy of celecoxib for pain management after arthroscopic surgery of hip: a prospective randomized placebo-controlled study. European Journal of Orthopaedic Surgery \& Traumatology 24: 919-923.

7. Schroeder KM, Donnelly MJ, Anderson BM, Ford MP, Keene JS (2013) The Analgesic Impact of Preoperative Lumbar Plexus Blocks for Hip Arthroscopy. A Retrospective Review. HIP International 23: 93-98. [crossref]

8. YaDeau JT, Tedore T, Goytizolo EA, et al. (2012) Lumbar plexus blockade reduces pain after hip arthroscopy: a prospective randomized controlled trial. Anesthesia and Analgesia 115: 968-972.

9. Dold AP, Murnaghan L, Xing J, et al. (2014) Preoperative Femoral Nerve Block in Hip Arthroscopic Surgery: A Retrospective Review of 108 Consecutive Cases. The American Journal of Sports Medicine 144-149.

10. Xing JG, Abdallah FW, Brull R, Oldfield S, Dold A, et al. (2015) Preoperative Femoral Nerve Block for Hip Arthroscopy: A Randomized, Triple Masked Controlled Trial. The American Journal of Sports Medicine 43: 2680-2687. [crossref]

11. Ward JP, Albert DB, Altman R, et al. (2012) Are Femoral Nerve Blocks Effective for Early Postoperative Pain Management after Hip Arthroscopy? Arthroscopy: The Journal of Arthroscopic and Related Surgery 28: 1064-1069.

12. Purcell RL, Nappo KE, Griffin DW, McCabe M, Anderson T, et al. (2018) Fascia iliaca blockade with the addition of liposomal bupivacaine vs. plain bupivacaine for perioperative pain management following hip arthroscopy. Knee Surgery Sports Traumatology Arthroscopy 26: 2536-2541. [crossref]
13. Stein BE, Srikumaran U, Tan EW, Freehill MT, Wilckens JH (2012) Lower-Extremity Peripheral Nerve Blocks in the Perioperative Pain Management of Orthopaedic Patients. J Bone Joint Surg Am 94: e167. [crossref]

14. Childs S, Pyne S, Nandra K, et al. (2017) The Effect of Intra-articular Cocktail versus Femoral Nerve Block for Patients Undergoing Hip Arthroscopy. Arthroscopy 33: 2170-2176.

15. Louw A, Diener I, Butler DS, Puentedura EJ (2013) Preoperative Education Addressing Postoperative Pain in Total Joint Arthroplasty: Review of Content and Educational Delivery Methods. Physiother Theory Pract 29: 175-194. [crossref]

16. Cogan CJ, Knesek M, Tjong VK, et al. (2016) Assessment of Intraoperative Intraarticular Morphine and Clonidine Injection in the Acute Postoperative Period After Hip Arthroscopy. Orthopaedic Journal of Sports Medicine 4: 2325967116631335.

17. Philippi MT, Kahn TL, Adeyemi TF, Nair R, Kahlenberg C, et al. (2018) Extracapsular local infiltration analgesia in hip arthroscopy: a retrospective study. Journal of Hip Preservation Surgery 5: 60-65. [crossref]

18. Garner M, MSc, Alsheemeri Z, MRCS, Sardesai, et al. (2016) A Prospective Randomized Controlled Trial Comparing the Efficacy of Fascia Iliaca Compartment Block Versus Local Anesthetic Infiltration After Hip Arthroscopic Surgery. Arthroscopy: The Journal of Arthroscopic and Related Surgery 33: 125-132.

19. Baker JF, McGuire CM, Byrne DP, Hunter K, Eustace N, et al. (2011) Analgesic control after hip arthroscopy: a randomised, double-blinded trial comparing portal with intra-articular infiltration of bupivacaine. Hip international: the journal of Clinical and Experimental Research on Hip Pathology and Therapy 21: 373-377. [crossref]

\section{Citation:}

LaPorte C, Rahl MD, Ayeni OR, Menge TJ (2021) Commentary: Postoperative Pain Management Strategies in Hip Arthroscopy. Integr J Anesth Sur Volume 2(1): 1-3. 